\title{
MEMS-CONTROLLABLE MICROLENS ARRAY FOR BEAM STEERING AND PRECISION ALIGNMENT IN OPTICAL INTERCONNECT SYSTEMS
}

\author{
Adisorn Tuantranont, Victor M. Bright, Jianglong Zhang, Wenge Zhang, John Neff, and Y. C. Lee
}

NSF Center for Advanced Manufacturing and Packaging of Microwave, Optical, and Digital Electronics (CAMPmode), Department of Mechanical Engineering, University of Colorado, Boulder, CO, USA 80309-0427 http://mems.colorado.edu, Tel: (303) 735-1734, Fax: (303) 492-3498,email:Adisorn.Tuantranont@colorado.edu

\section{ABSTRACT}

Novel, 2-dimensional MEMS-controllable microlens array has been integrated with a Vertical-Cavity-Surface-Emitting-Laser (VCSEL) array using a flip-chip assembly. The MEMS/VCSEL hybrid system is demonstrated for efficient and reliable smart pixel arrays for board-to-board or chip-to-chip optical interconnects in digital systems. By translating polymer microlens, fabricated on a MEMS X-Y movable plate, using electro-thermal actuators, a beam steering angle of $70 \mathrm{mrad}$ is achieved. VCSEL beam steering was successfully demonstrated in our MEMS/VCSEL hybrid system to collimate and steer laser beam for a precision alignment in a 2-dimensional free-space optical interconnect.

\section{INTRODUCTION}

Free-space optical interconnection is attractive for several applications including telecommunication switching networks and fine-grained parallel processing /computers. Currently optical interconnects are used for data communication over long and medium distances in wide area networks (WANs). However over short distances inside computer systems or clusters of electronic networks, board-to-board or chip-to-chip multi-GHz bandwidth optical interconnects are required to replace electrical interconnects in the near future. This replacement is necessary due to problems with signal integrity and crosstalk at high frequency in electrical interconnects. The advantages of free-space holographic interconnects include direct interconnects between boards, arbitrary interconnection patterns, multiple fan-outs, channel isolation and increased bandwidth, thus avoiding the interconnection bandwidth bottleneck of systems with strictly inplane electronic interconnects. Furthermore, the cost of free-space interconnects

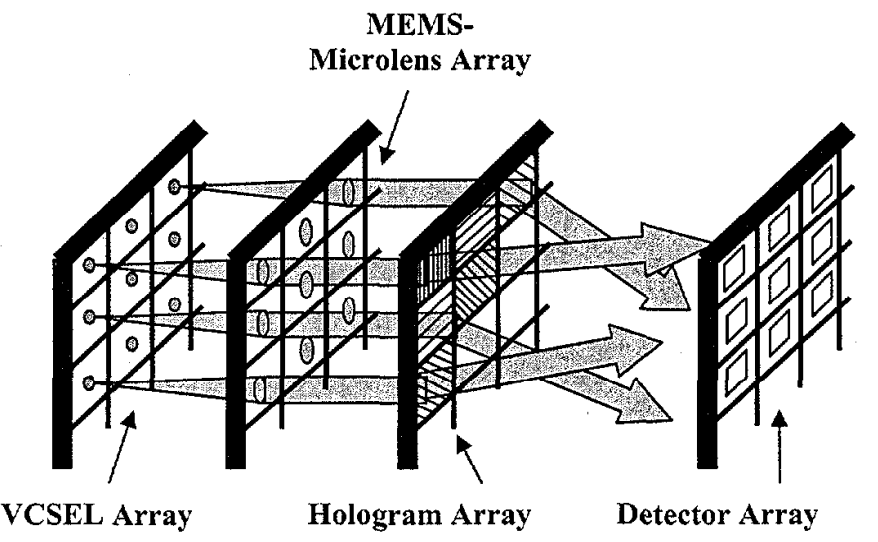

Figure 1. MEMS-controllable microlens array for enhanced alignment in a 2-dimensianal board-to-board optical interconnect. has been reduced substantially with the advancement of verticalcavity surface-emitting lasers (VCSELs). However alignment challenges are inevitable in optical interconnect systems. Mechanical vibrations or thermal expansion can cause misalignment in the systems. In this paper, we propose an enhanced alignment approach for free-spacc interconnects without a demand for tighter assembly tolerances using a MEMScontrollable microlens array. Figure 1 shows the use of MEMScontrollable microlens array to enhance alignment for efficient and reliable smart pixel arrays on board-to-board optical interconnect in digital systems. The microlens collimates an incident VCSEL beam and the microlens actuators steer the beam to a predefined position on a hologram array by laterally translating the microlens. Several groups have recently described efforts to steer laser beam using decentered microlens techniques [1-2]. These approaches require a light-weight and small travel of the microlens components to achieve agile beam steering. Despite the number of papers on decentered microlens beam steering, all of them seem to require a large space for array of actuators such as comb-drive actuators or scratch drive actuators (SDA) to laterally translate microlens. Moreover, none of them has operated at a VCSEL wavelength of $830 \mathrm{~nm}$ because the MEMS devices need to be fabricated on a silicon substrate, which is not transparent at this wavelength. The device presented in our approach contains a dense array of individually controllable microlenses, allowing for operation with a VCSEL array. In this paper, the design, fabrication, MEMS/NCSEL integration, and beam steering results are presented.

\section{MEMS-CONTROLLABLE MICROLENS}

The 2-dimensional MEMS-controllable microlens array was fabricated through the commercially available surface micromachining technology (Multi-User MEMS Process-MUMPs) [3]. Photo-bisbenzocyclobutene (BCB) electronic resin, a photosensitive polymer, was used in our own process to fabricate micro semi-spherical polymer lenses on MEMS. Each $52.5 \mu \mathrm{m}$ focal length microlens was fabricated directly on a MEMS X-Y movable plate with a $30 \mu \mathrm{m}$ circular aperture as shown in Figure 2. The microlens was modeled as the thin paraxial lens. The focal length of the microlens is set by controlling the initial thickness of the photo-BCB layer and the diameter of the lens, which are defined by photolithography. The photo-BCB thickness required for a given radius of curvature for the lens was calculated by equating the volume of spherical portion representing the final lens shape to the volume of a cylinder representing the photo-BCB column before reflow [4]. Transmission of microlens is more than $95 \%$ at operating wavelength $(830 \mathrm{~nm})$. The lens profile is in $5 \%$ error compared to an ideal spherical lens profile. Since only small fraction of lens surface is used to refract light, the effects of spherical aberration and steering-angle-dependent off-axis wavefront aberration are minimized. 


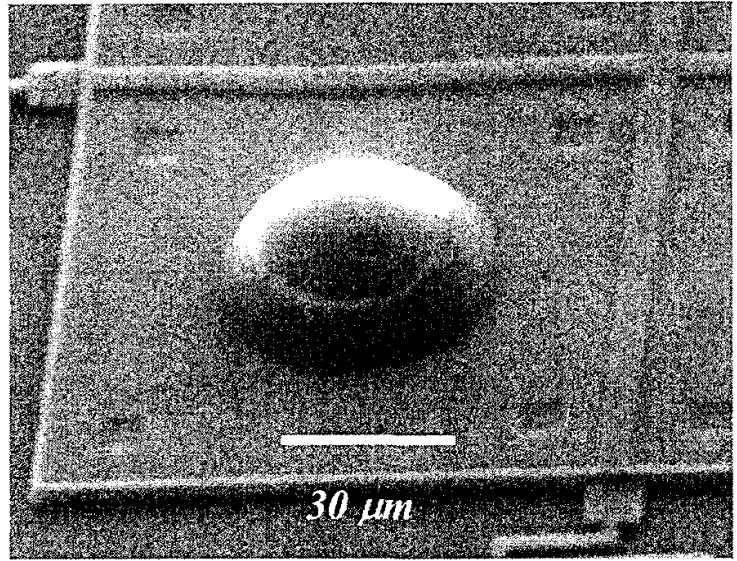

Figure 2. Polymer microlens fabricated on a polysilicon MEMS movable plate.

Two arrays of electro-thermal actuators are coupled with the $80 \mu \mathrm{m} \times 80 \mu \mathrm{m}$ polysilicon movable plate to translate the microlens in X-Y plane. The microlens array is located at a focal plane in front of the VCSEL array and collimates the incident VCSEL beams. By the concept of decentered microlens, if the microlens is decentered with respect to the beam axis, then the beam will propagate through the off-axis point of microlens. The microlens still collimates the beam, but the beam is directed to a nonzero field angle as shown in Figure 3.

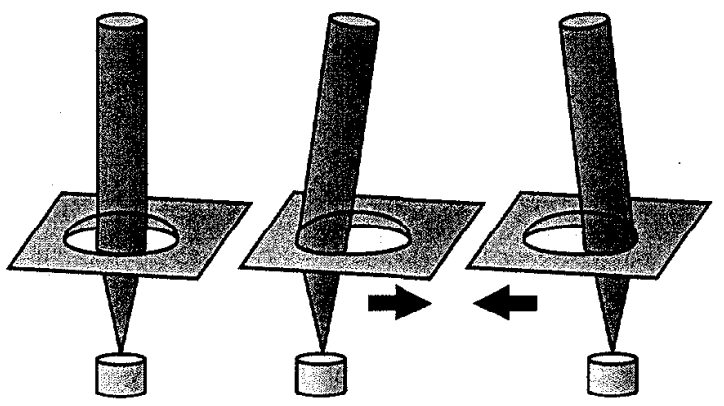

Figure 3. The decentered microlens concept of MEMScontrollable microlens array.

The steering angle depends on the lateral displacement $\Delta d$ of lens with respect to the beam axis and the focal length $f$ of the lens as shown in Figure 4. In this scheme, large steering angles are possible by large lens displacement on the order of lens radius.

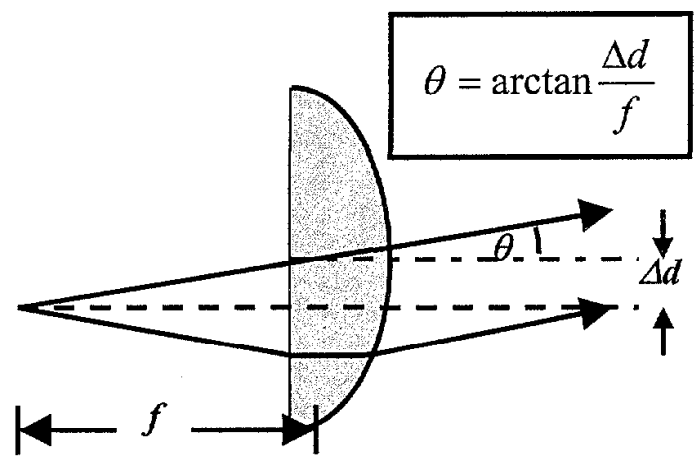

Figure 4. The steering angle of a decentered microlens.
A simulation of the MEMS-controllable microlens was conducted with ABAQUS, which is a commercially available finite element analysis tool. The model consists of an electrothermal analysis to obtain the temperature distribution resulting from a simulated input power to electro-thermal actuators. This is then coupled to mechanical analysis in which the temperature distribution is used to determine deflections resulting from thermal expansion mismatch in hot/cold arms of actuators. The finite element simulation of the temperature distribution in actuators and movement of the plate is shown in Figure 5.
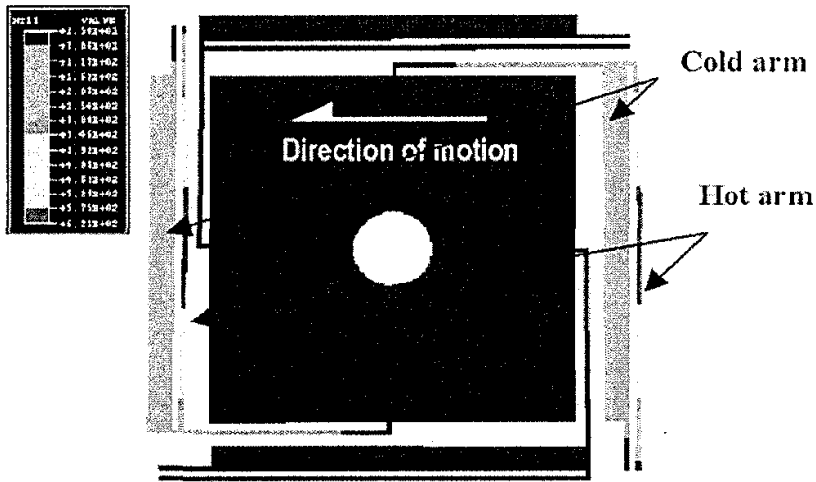

Figure 5. FEM simulation of thermal distribution and movement in electro-thermal actuators.

' $U$ '-shaped lateral electro-thermal actuators or 'heatuators' used to drive central plate in a lateral motion consist of a singlematerial connected narrow arm and wider arm. Current is passed through the polysilicon actuator, and the higher current density in the narrower 'hot' arm causes it to heat and expand more than the wider 'cold' arm. The arms are joined at the free end, which constrains the actuator tip to move laterally in an arcing motion towards the cold arm side [5]. The tips of the actuators are coupled to the central plate by long thin flexures on opposite sides of the plate. Left actuator in Figure 5 pulls the plate while the right actuator pushes the plate at the same time. By push/pull mechanism of actuators, the motion of the plate is linearly proportional to the consumed power. The mechanical crosstalk in $\mathrm{X}$ and $\mathrm{Y}$ directions is minimized by using long thin flexures.

\section{MEMS FLIP CHIP TRANSFER/INTEGRATION}

The procedures for flip-chip transfer of MEMS to a nonsilicon substrate (in this case quartz substrate) need two postprocessing steps [6]. First, the MEMS structures are fabricated at a commercial foundry as shown in Figure 6(a), where a MEMS device is attached to the silicon substrate only by the oxide encasing it. The target substrate is patterned with gold interconnects and bonding pads designed to receive the MEMS structures, as shown in Figure 6(b). Then, in the second step shown in Figure 6(c), the two chips are bonded together by thermosonic bonding. Finally, the bonded structure is subjected to a hydrofluoric acid (HF) rinse, as shown in Figure 6(d), to free the MEMS structure and remove the silicon substrate.

The MEMS-controllable microlens array was designed and patterned in the first releasable polysilicon layer of the MUMPs process. The photo-BCB microlenses are patterned and fabricated on polysilicon plates by our own process. The plates and actuators are built upside-down on top of sacrificial oxide so that the bonding pads fabricated on MEMS are matched with bonding pads 
Oxide layer

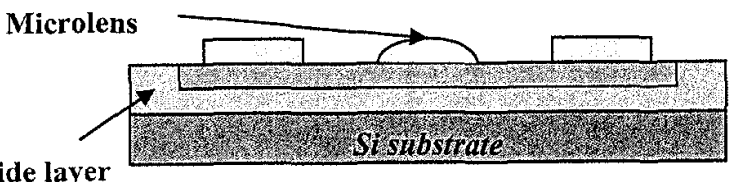

(a) MEMS structure with microlens on silicon substrate.

Gold bump

Transparent substrate

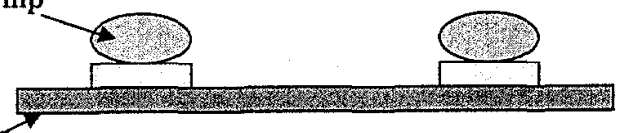

(b) Matching gold bonding pads and interconnects.

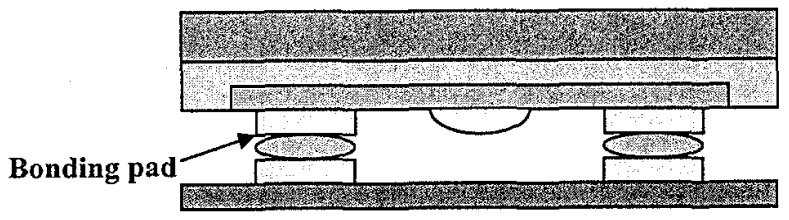

(c) Flip chip and thermosonic bonding.

MEMS

structure

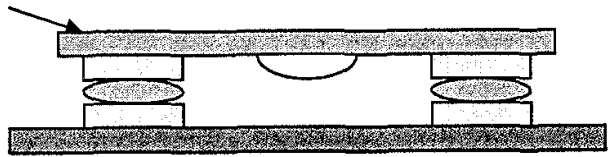

(d) Release of MEMS structure from silicon substrate.

Figure 6. Flip-chip assembly process of MEMS to a non-silicon substrate.

on quartz target substrate. The quartz receiving substrate was patterned with gold wires and bonding pads for flip-chip bonding and wire bonding. A layer of photorcsist is used to protect the quartz and gold interconnects when the assembly is released in HF. The layer is patterned to remove the photoresist over the bonding pads to expose the pads for flip-chip bonding. Gold bumps of 70 $\mu \mathrm{m}$ height are placed on pads and used to bond and electrically connect the MEMS structure and the wiring substrate. The MEMScontrollable microlens array is then flipped and bonded with the wiring quartz substrate using thermosonic bonding. The final step in process is to release the bonded device in a HF bath and remove the silicon substrate. The final device after flip-chip transfer to a quartz substrate is shown in Figure 7.

\section{VCSEL ARRAY}

The VCSEL array consists of an $8 \times 8$ array of top-emitting VCSELs on a $250 \mu \mathrm{m}$ pitch. Molecular Beam Epitaxy (MBE) was used to grow the VCSEL structurc on a GaAs substrate. The VCSEL structure consists of two distributed Bragg reflector (DBF) mirrors surrounding a single GaAs quantum well, and is ion implanted for current confinement in the active region. The VCSEL operates at approximately $830 \mathrm{~nm}$ with a bandwidth of 0.2 $\mathrm{nm}$ and a $2 \mathrm{~nm}$ wavelength variation across the array. A Guassian beam with a $\left(e^{-2}\right)$ half-angle of 0.135 radians ( $2 \mu \mathrm{m}$ in waist) is emitted from the top surface [7]. The threshold current is $1.5 \mathrm{~mA}$. The VCSEL array is flip-chip bonded on a glass substrate using thermosonic bonding. The bonding process uses gold or gold-tin alloy, which is applied to the contacts. The package containing the VCSEL is soldered to a carrier board. A commercially available microlens array, which has a focal length of $560 \mu \mathrm{m}$ and a pitch of

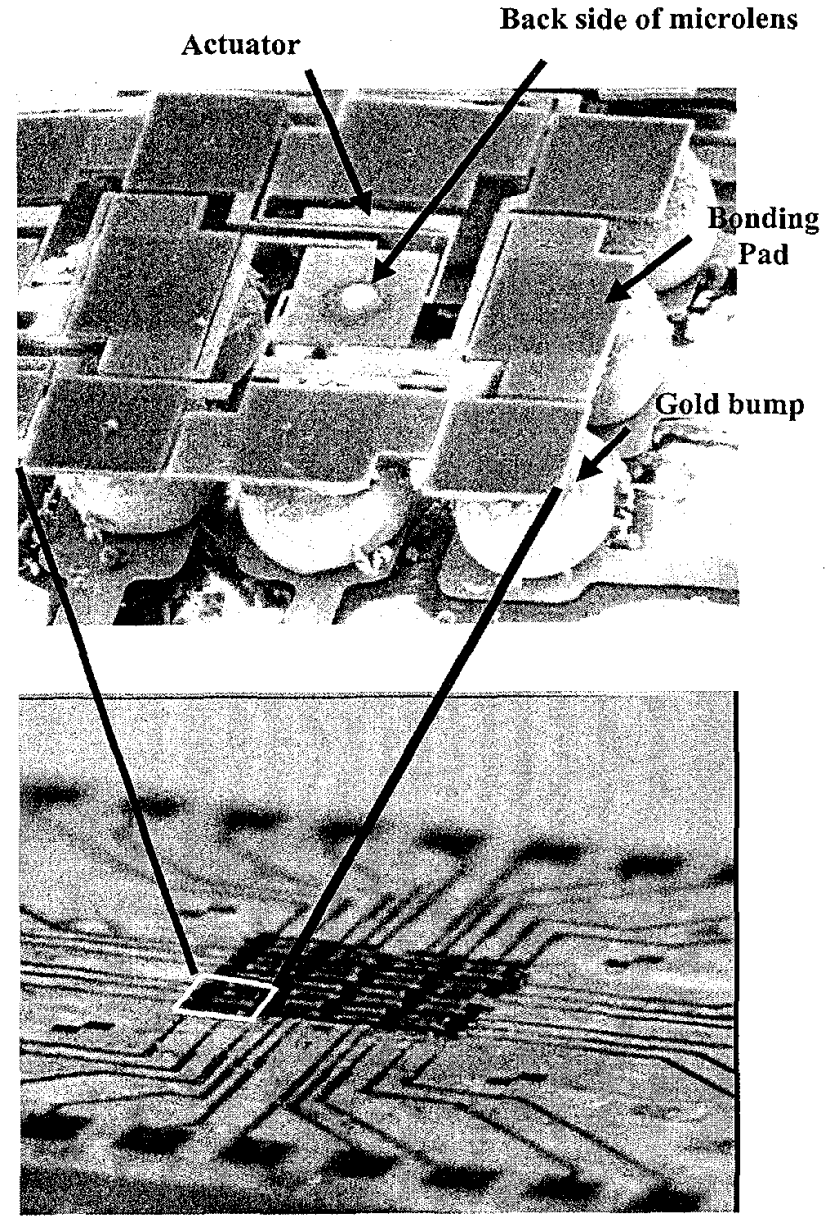

Figure 7. SEMs of the flip-chip assembled MEMS-controllable microlens $4 \times 4$ array on a quartz substrate.

$250 \mu \mathrm{m}$ is aligned with VCSEL array and attached to the opposite side of the glass substrate using a UV-curable epoxy. The resultant collimated beams are $160 \mu \mathrm{m}$ in diameter. Figure 8 shows a packaged VCSEL array.

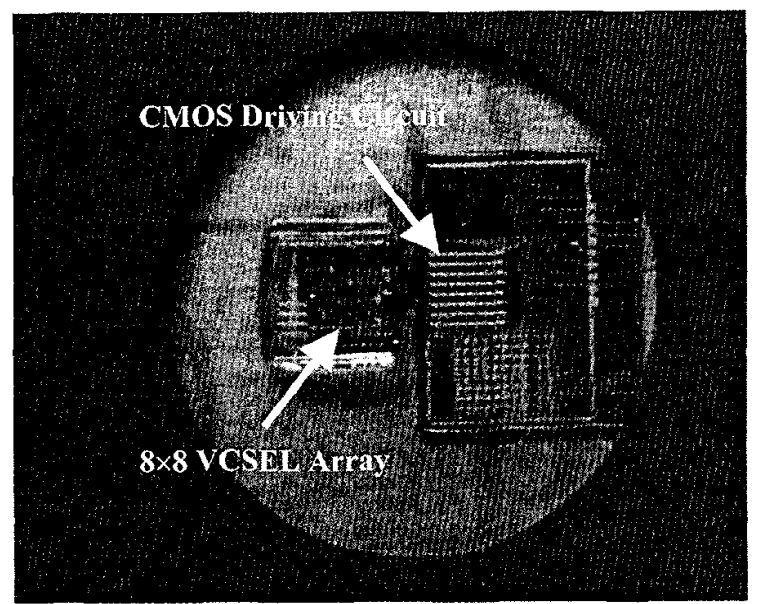

Figure 8. Flip-chip assembled $8 \times 8$ VCSEL array on a glass substrate. 


\section{BEAM STEERING RESULTS}

The MEMS-controllable microlens array chip was actively aligned with the VCSEL array chip. First, a decentered keplerian telescope is used to achieve a collimated beam steering. The controllable microlens is placed at its focal length $f_{M}$ in front of the focal plane of the lens $f_{L}$. The microlens then collimates and steers beam to the disired directions by translating in $X-Y$ plane. Consequently, the telescope optics, which consist of positive lens $\left(f_{1}=100 \mathrm{~mm}\right)$ and negative lens $\left(f_{2}=-50 \mathrm{~mm}\right)$ are used to magnify the steering angle by the ratio of $f_{1} / f_{2}=2$ ). CCD camera was used to detect the deflection of the beams in the far field plane. Figure 9 shows the keplerian telescope and telescope lenses in the beam steering experiment setup. The 2-dimensional beam steering is successfully demonstrated as shown in Figure 10.

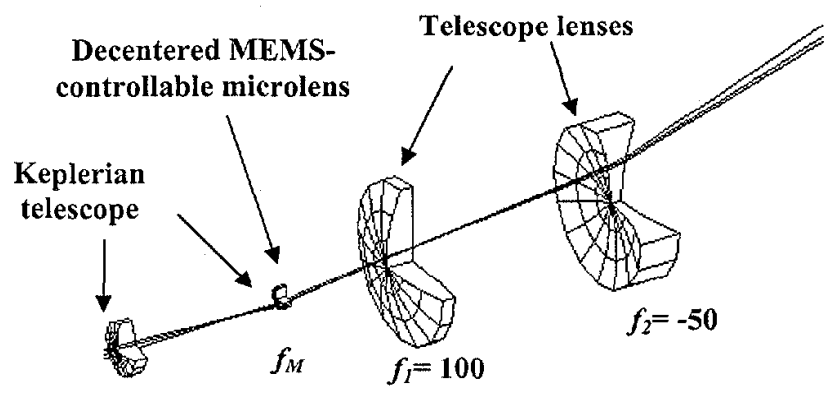

$f_{L}=25$

$\underline{\text { Units in mm. }}$

Figure 9. Optical experimental setup for beam steering demonstration.

The maximum beam steering of $70 \mathrm{mrad}(\approx 4$ degrees) is implemented by translating the microlens by one-half of the lenslet diameter as shown in Figure 11, which is adequate for enhancing optical alignment in free-space optical interconnects. Measurement verifies insertion loss of $<2 \mathrm{~dB}$. The system was demonstrated up to a rate of $1 \mathrm{kHz}$ of operation frequency, result of the low inertia and small travel of the microlens components.

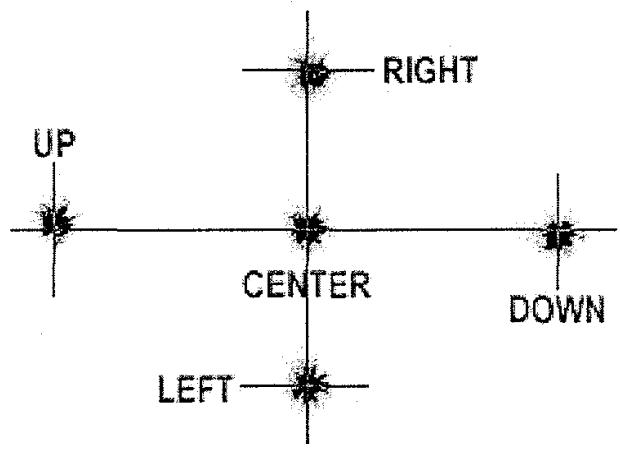

Figure 10. The 2-dimensionat beam steering demonstration.

\section{CONCLUSIONS}

Novel, 2-dimensional MFMS-controllable microlens array has been integrated with a Vertical-Cavity-Surface-Emitting-Laser (VCSEL) array. Flip-chip transfer of MEMS structure on transparent substrate is described in detail. By translating polymer microlens fabricated on MEMS X-Y movable plate using electrothermal actuators, the 2-dimensional bcam steering is demonstrated

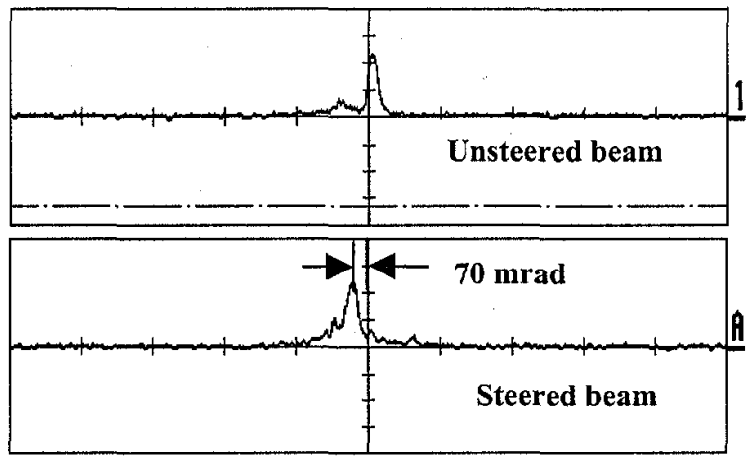

Figure 11. The maximum beam steering angle of 70 milliradians.

and the maximum beam steering of $70 \mathrm{mrad}$ is achieved. VCSEL beam steering was successfully demonstrated in our MEMS/VCSEL hybrid system to collimate and steer laser beam for a precision alignment in a 2-dimensional free-space optical interconnect system.

\section{ACKNOWLEDGEMENTS}

This work is supported by the U.S. Air Force Office of Scientific Research (AFOSR), Grant\#F49620-98-1-0291. Also thanks to V. Thiantamrong, Li-Anne Liew, and Kevin Harsh.

\section{REFERENCES}

1. H. Toshiyoshi, J. G. Su, J. Lacosse, and M. C. Wu, "Micromechanical lens scanners for fiber optics switches", Proc. MOEMS'99, pp. 165-170 (1999).

2. R. Goring, D. Doring, P. Bucker, B. Gotz, P. Chreiber, P. Dannberg, E. B. Kley, and M. Cumme, "Microoptical concepts for miniaturised scanners and switches from design to realisation", Proc. MOEMS'99, pp. 76-87 (1999).

3. D. A. Koester, R. Mahadevan, A. Shishkoff, and K. W. Markus, "Multi-User MEMS Processes (MUMPs): Design handbook, Rev. 4," Cronos Integrated Microsystems, 3021 Cornwallis Road, Research Triangle Park, NC 27709 (1999).

4. A. Tuantranont, V. M. Bright, W. Zhang, J. Zhang, and Y. C. Lee, "Self-aligned assembly of microlens arrays with micromirrors," SPIE Vol. 3878, pp. 90-101 (1999).

5. J. H. Comtois and V. M. Bright, "Surface micromachined polysilicon thermal actuator arrays and applications", Technical Digest of the 1996 Solid-State Sensor and Actuator Workshop, Hilton Head Isl., SC, pp. 174-177.

6. W. Zhang, K. F. Harsh, M. A. Michalicek, V. M. Bright, and Y. C. Lee, "Flip-chip assembly for RF and oplical MEMS', Proc. ASME InterPack'99, Vol. EEP-26-1, pp. 349-354 (1999).

7. H. J. Zhou, J. Neff, Y. Chen, V. Fedor, Y. C. Lee, C. C. Mou, W. Berseth, T. Mclaren, and E. Tang, "Demonstration of a massively parallel bi-directional crosspoint switch with optical control", SPIE Vol. 3005, pp. 266-272 (1997). 